\title{
Plea for an international Aneurysm Data Bank: description and perspectives
}

\author{
Philippe Bijlenga, MD, PhD, ${ }^{1}$ Sandrine Morel, PhD, ${ }^{1,2}$ Sven Hirsch, PhD, ${ }^{3}$ Karl Schaller, MD, ${ }^{1}$ and \\ Daniel Rüfenacht, MD4
}

${ }^{1}$ Neurosurgery Division, Department of Clinical Neurosciences, Faculty of Medicine, Geneva University Medical Center, Geneva; ${ }^{2}$ Department of Pathology and Immunology, University of Geneva, Faculty of Medicine, Geneva; ${ }^{3}$ Institute of Applied Simulation, University of Applied Sciences, Wädenswil, Zürich; and ${ }^{4}$ Neuroradiologie, SwissNeurolnstitute, Klinik Hirslanden, Zürich, Switzerland

The disease resulting in the formation, growth, and rupture of intracranial aneurysms is complex. Research is accumulating evidence that the disease is driven by many different factors, some constant and others variable over time. Combinations of factors may induce specific biophysical reactions at different stages of the disease. A better understanding of the biophysical mechanisms responsible for the disease initiation and progression is essential to predict the natural history of the disease. More accurate predictions are mandatory to adequately balance risks between observation and intervention at the individual level as expected in the age of personalized medicine. Multidisciplinary exploration of the disease also opens an avenue to the discovery of possible preventive actions or medical treatments. Modern information technologies and data processing methods offer tools to address such complex challenges requiring 1) the collection of a high volume of information provided globally, 2) integration and harmonization of the information, and 3) management of data sharing with a broad spectrum of stakeholders.

Over the last decade an infrastructure has been set up and is now made available to the academic community to support and promote exploration of intracranial disease, modeling, and clinical management simulation and monitoring.

The background and purpose of the infrastructure is reviewed. The infrastructure data flow architecture is presented. The basic concepts of disease modeling that oriented the design of the core information model are explained. Disease phases, milestones, cases stratification group in each phase, key relevant factors, and outcomes are defined. Data processing and disease model visualization tools are presented. Most relevant contributions to the literature resulting from the exploitation of the infrastructure are reviewed, and future perspectives are discussed.

https://thejns.org/doi/abs/10.3171/2019.4.FOCUS19185

KEYWORDS disease modeling; data integration; data sharing; data management

$\mathrm{C}$ LINICIANS providing care to patients newly diagnosed with any condition are exposed to a multitude of questions. The answers to those questions may be based on robust knowledge and evidence, widely disseminated in the medical community and well accepted, while others may be disputed, based on new emerging notions and a limited quantity of data that may be contradictory or biased. Adequately answering questions and making decisions by making the most of the latest evidence and knowledge relevant to a particular patient is a major challenge.
When counseling patients diagnosed with intracranial aneurysms, the first questions are typically as follows: Is this aneurysm potentially dangerous? How will it evolve? Is treatment possible? What are the risks associated with treatments? Are there any recommendations for a change in lifestyle? Should other family members be examined? All of these questions have slightly different answers depending on factors specific to each patient. The management of patients with intracranial aneurysms is not limited to patients diagnosed with an aneurysm incidentally, but also extends to patients with symptoms related to an aneu-

ABBREVIATIONS aSAH = aneurysmal SAH; ADB = Aneurysm Data Bank; CRIM = Clinical Reference Information Model; ISUIA = International Study of Unruptured Intracranial Aneurysms; $\mathrm{SAH}=$ subarachnoid hemorrhage; UIA = unruptured intracranial aneurysm.

SUBMITTED February 28, 2019. ACCEPTED April 24, 2019.

INCLUDE WHEN CITING DOI: 10.3171/2019.4.FOCUS19185. 
rysm or having suffered from a rupture of the aneurysm. This corresponds to different phases of the disease and, for each phase of the disease, the answers depend on a small number of severity factors, variable factors, and modifiable factors. Since the long-term prognosis of patients is influenced by a multitude of decisions made during different phases of illness and life, it has a high potential for optimization.

Patients incidentally diagnosed with an intracranial aneurysm were traditionally informed that the lesion might rupture and destroy their life like a bomb in the head. This frightening language has prompted many patients to be treated aggressively at the risk of suffering the collateral damage of a risky intervention. The study of the International Study of Unruptured Intracranial Aneurysms (ISUIA) ${ }^{18}$ suggested that some aneurysms did not expose patients to such a high risk and suggested that in some cases the risks associated with interventions could exceed the risks associated with natural evolution.

The assessment of the risk of aneurysm rupture is difficult because it is based on at least 6 factors and their combinations, namely, the type of vascular lesion being saccular or fusiform; the location of the aneurysm in the cerebrovascular tree; the size of the aneurysm sac; and the patient's age, sex, and family history. If these factors were dichotomous, their various combinations would already generate 720 different categories, but some of these factors are categorial or continuums. In addition, according to a consensus study, the list of relevant factors to be taken into account may be much greater. Indeed, the UIATS (unruptured intracranial aneurysm treatment score) system in which points are summed in favor of or against an aggressive treatment is based on 27 factors.

The risk associated with treatment of intracranial aneurysms in general is less than $0.5 \%$ mortality and less than $5 \%$ morbidity but rises to $22 \%$ mortality and $32 \%$ morbidity in high-risk groups. ${ }^{8,11-13}$ This risk is to be weighed against the risk associated with the potential rupture of the aneurysm. Often, the difference regarding risks associated with different options are small. The quality and the gradual improvement of care through the development of multiple innovations reduces risks of interventions and often simplifying decision-making in favor of active treatment. Nevertheless, distinguishing the best option when differences become smaller requires larger cohorts and multicenter and international collaboration. Since most patients are asymptomatic when initially diagnosed, the choice of treatments and consequences of failure become extremely relevant. It is essential to determine an acceptable level of performance and develop tools to improve efficiency.

At present, the possibility of assessing the efficacy of one treatment over another is limited by the feasibility of a study requiring the rapid recruitment of a large number of patients and therefore the establishment of a heavy and expensive structure. The rapidity of technological developments, the strong competition, and the obligation to ensure safety but not to demonstrate the superiority of new treatments, encourages the medical community to respond to the satisfaction of stakeholders or patients but not to systematically commit resources to effectively measure treatment performance.
It is estimated that $3 \%$ of the population suffers from an intracranial aneurysm and thus there is a potential major impact of each decision during care. Addressing the issue is a question of public health and, due to the disease complexity and diversity of care options, it is an illustrative example of the challenge of personalized medicine. The additional challenge is to manage collaboration, systematic analysis, and harmonized documentation of care for a high number of hospitals globally.

New technologies offer a fantastic opportunity to federate, harmonize, integrate, and contextualize information. To meet the challenge of personalized medicine, we propose here an evolutionary infrastructure for the massive collection of clinical and radiological data and their harmonization, allowing on the one hand the acquisition of a sufficient statistical power to carry out clinical studies, and on the other hand providing a decision aid for the management of intracranial aneurysms. Only large-scale collections of high-quality longitudinal data will meet the challenge of personalized medicine.

\section{Infrastructure of the Aneurysm Data Bank}

The development of a data bank requires generic components that apply to all aspects of health and specific components applying to particular organs and pathologies. This infrastructure must ensure the exchange of health information, measures taken for the maintenance of health, treatments and interventions and their consequences, and development of structures regulating the flow and exploitation of information.

The development of the infrastructure described here was initiated as part of the calls for tender of the 6th Framework Program for Research and Innovation of the European Commission (FP6), for which the project "@neurIST: integrated bioinformatics for the management of intracranial aneurysms" had been funded (20062010, www.aneurist.org). Since 2015, the development of @ neurIST continued in the framework of the project "AneuX: shape as a biomarker for aneurysm disease" funded by the Swiss initiative for systems biology (SystemsX.ch; http://www.systemsx.ch/fr/projets/medical-research-anddevelopment-projects/aneux/).

At present, the infrastructure hosts data for 1) patients diagnosed with one or more intracranial aneurysms, family members, and healthy volunteers recruited at Geneva University Hospitals since 2006 according to the @ neurIST protocol and Geneva Ethics authorization CCER 07-056 and PB_2018-00073; 2) participants recruited under the @ neurIST project between 2006 and 2010 in partner centers in Barcelona (Spain), Amsterdam (the Netherlands), Pecs (Hungary), and Oxford and Sheffield (United Kingdom); and 3) participants recruited for studies evaluating the genetic predisposition to the formation of intracranial aneurysms and federated by the International Stroke Genetics Consortium (ISGC). Data from more than 7000 participants have been harmonized and hosted by this infrastructure. Digital data represent genetic, biological, structural, and functional information corresponding to the various phases of patient diagnosis, management, treatments, and interventions as well as the 
evolution of the disease. All of these data are associated and harmonized using a disease-specific Clinical Reference Information Model (CRIM). The CRIM has been improved continuously up to current version 5 , which is compatible with the format defined for Unruptured Intracranial Aneurysms and Subarachnoid Hemorrhage by the NIH Common Data Elements working groups. ${ }^{4}$

From a technical point of view, this structure is divided into 3 main modules (Fig. 1), allowing 1) data acquisition (care zone); 2) standardization and management of data sharing (data sharing infrastructure); and 3) exploitation of data for information or research (information/research zone).

\section{Database Design and Disease Modeling}

The data are labeled and stored to facilitate case-based lifelong recording of information relative to the initial patient condition, exposition to constant, variable, and modifiable factors, as well as outcomes, complications, and adverse events. Each case populates a disease model where the disease is divided into a succession of phases (Table 1). The phases are defined by milestones. Cases are clustered in severity groups specific to each phase. When multiple aneurysms are identified, both patients and aneurysms are grouped in severity groups. The milestones defining the phases are 1) the diagnosis of at least one intracranial an- eurysm, 2) the observation of a modification of the aneurysm or observation of a new aneurysm, 3) the treatment of an aneurysm, 4) the rupture of an aneurysm, 5) the diagnosis of vasospasm, 6) the diagnosis of hydrocephalus, 7) the diagnosis of a treatment failure, and 8) death.

The first phase is the premorbid condition prior to the diagnosis of an intracranial aneurysm. This phase stops the day a subject is diagnosed with an intracranial aneurysm and starts to be a patient. Patients are clustered in different groups as 1) those having no factors associated with the disease, 2) those having characteristics exposing them to the risk of intracranial aneurysm, and 3) those having characteristics exposing them to the risk of intracranial aneurysm, justifying a screening. It is expected that with growing knowledge regarding genetics and risk factors, subject classifiers will be developed, allowing the estimation of a lifelong probability of disease susceptibility or disease occurrence rate.

The second phase starts with the diagnosis of an intracranial aneurysm. Patients are currently classified in different groups according to the estimation of risk by clinicians. Patients can be diagnosed with unruptured intracranial aneurysms (UIAs) that are considered at low risk and observed with regular follow-ups $(F)$. The patients who undergo follow-up may have lesions that remain stable (S) or grow $(\mathrm{G})$. The definition of growth is broad and may change. Aneurysm growth has so far included the obvious

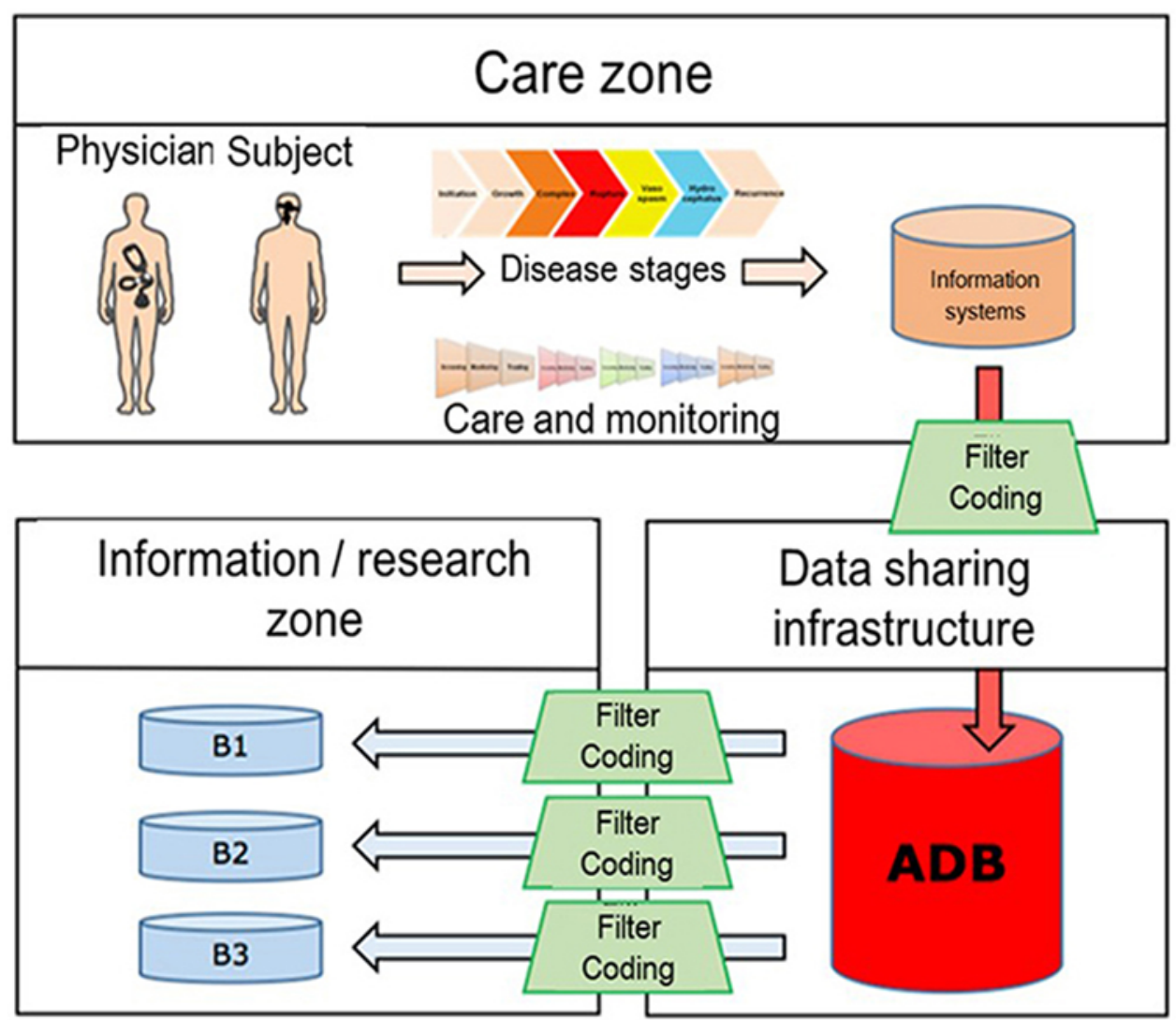

FIG. 1. The infrastructure provides different levels of decision support, promoting standardized data collection in the clinical environment. Data from different providers are harmonized and integrated to promote disease understanding, modeling, and management simulation by global information sharing. The data are packaged individually to data users. 


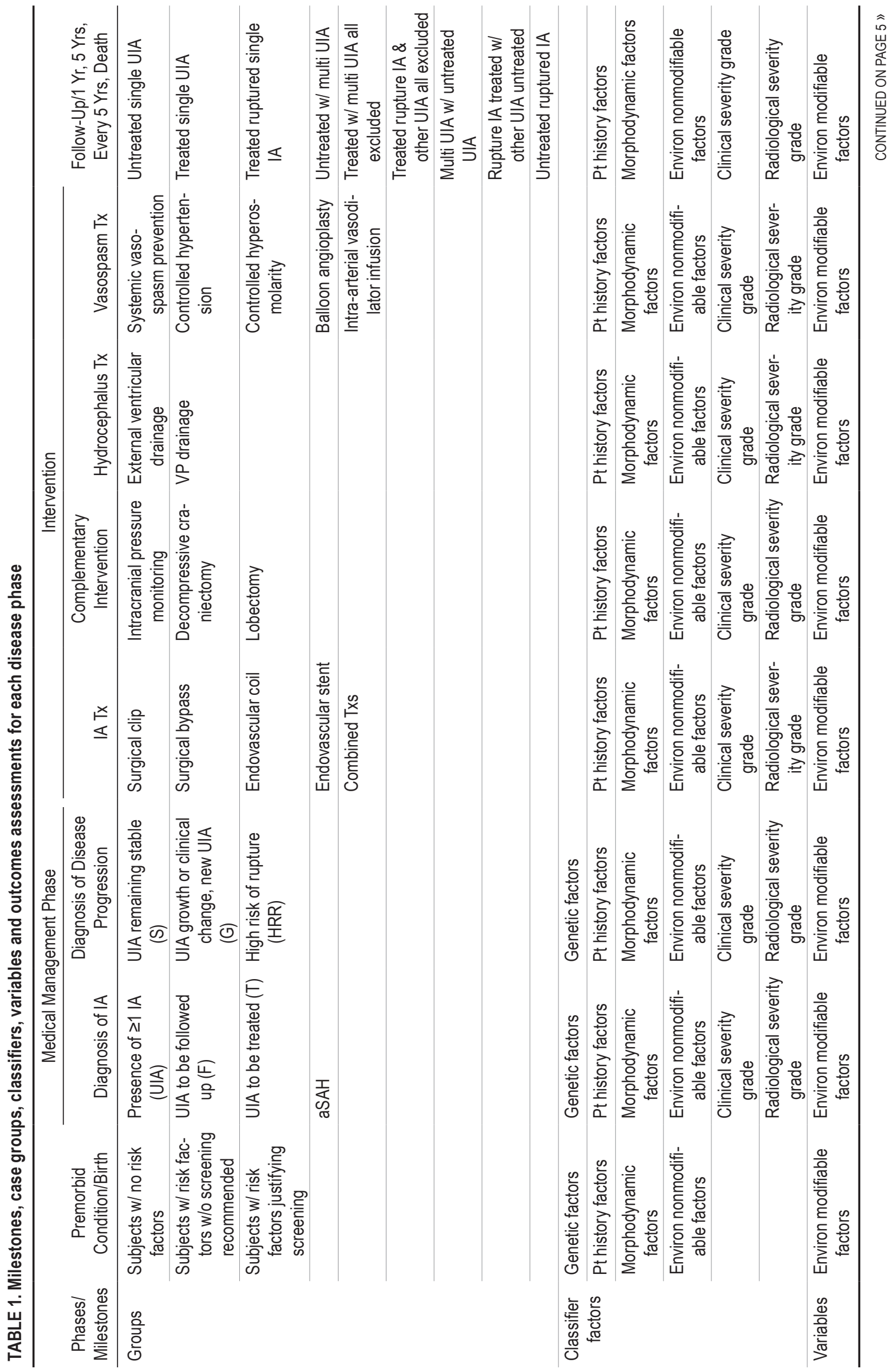




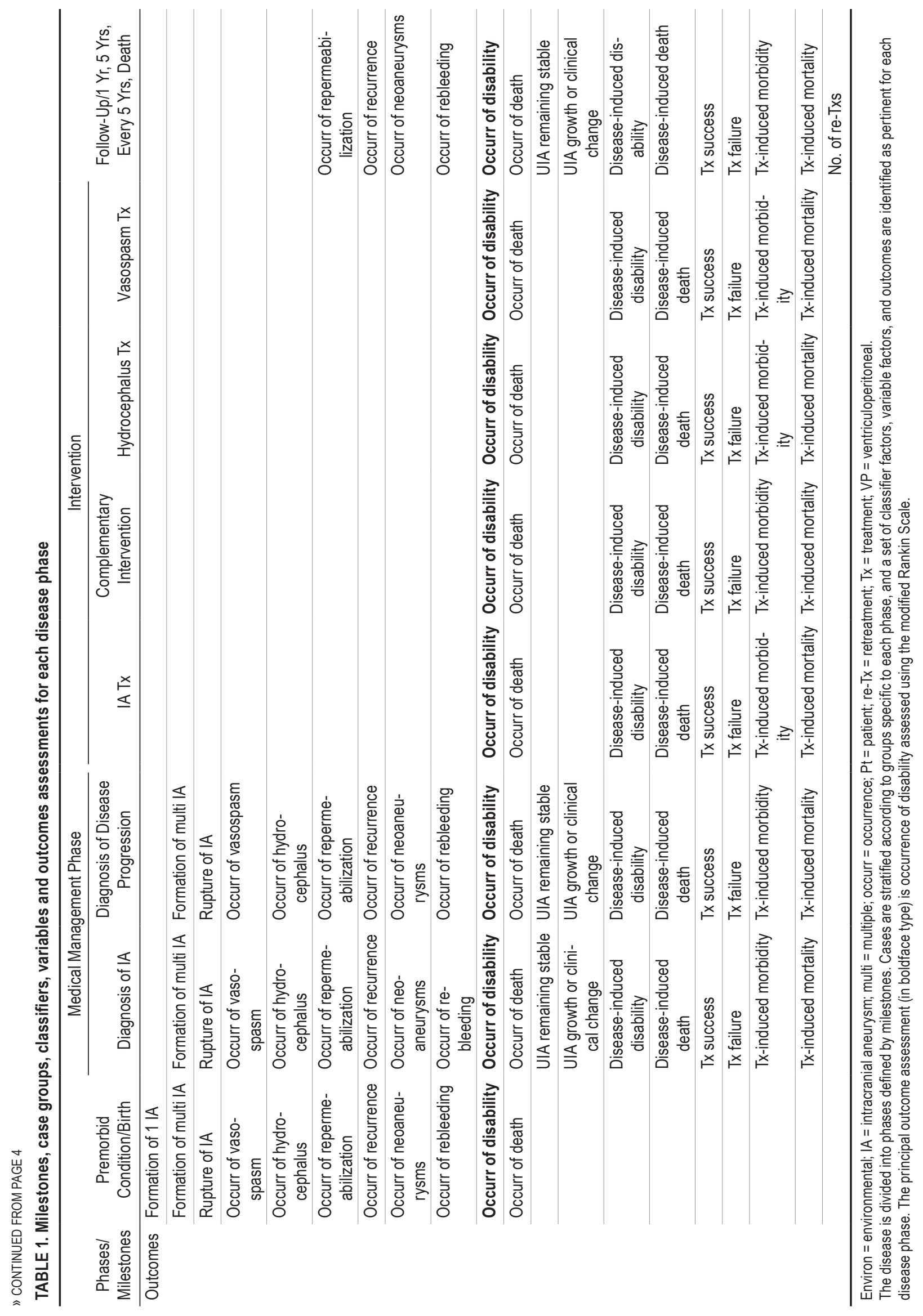


observation of morphological changes of the aneurysm dome but also changes in headaches or changes in aneurysm wall characteristics on imaging, leading to a change in the clinical recommendation and proceeding to an intervention (Gondar et al. ${ }^{10}$ ). A patient initially diagnosed with a UIA can be considered on the basis of clinical judgment of risk of aneurysm rupture and treated (T). Patients diagnosed with an intracranial aneurysm in the context of a subarachnoid hemorrhage ( $\mathrm{SAH}$ ) due to the rupture of the aneurysm populate the aneurysmal SAH (aSAH) group. Groups of patients in G, T, and aSAH can be pooled to create a high-rupture-risk patient group (HRR) that can be compared with patients with the lowest risk, diagnosed with stable aneurysms (S). The comparison of these 7 groups (UIA, F, S, G, T, aSAH, and HRR) allows the assessment of factors associated with or models to predict 1) the risk of aneurysm rupture (UIA vs aSAH), 2) rate of aneurysm growth or rupture using longitudinal data, 3) the probability of the UIA to be stable (S vs HRR), and 4) clinical decision making regarding selection of patients for observation versus treatment and different treatment modalities ( $\mathrm{F}$ vs $\mathrm{T}$ ).

The third phase starts with the first treatment of an aneurysm and records information regarding clinical management. It is divided into a great number of groups according to initial conditions collected in the first 2 phases and different treatment methods and sequences of treatment. It covers not only the treatment of aneurysms but also the management of epilepsy and stroke or consequences of SAH like vasospasm and hydrocephalus.

The fourth phase is capturing outcomes by assessing case evolution over time. The primary outcome is the quantification of disability integrated over a lifetime using the modified Rankin Scale assessed at 1 year and 5 years after initial diagnosis and every 5 years thereafter over the rest of the patient's life. Secondary outcome assessments are neurocognitive performance using the Montreal
Cognitive Assessment and quality of life using the SF-36 and potentially other emerging assessment tools, as well as treatment failures like aneurysm repermeabilization, recurrence, neoaneurysm formation, and rebleeding.

The disease model is progressively populated with harmonized data collected transversally and longitudinally and integrated from different previous and ongoing studies. Care is taken to populate the disease model also with data collected prospectively and consecutively for the direct purpose of disease model validation.

The disease model is progressively constructed by integrating successive modules, allowing the prediction of transitions from one milestone to another within the different phases. The prediction results of each module feed the classifiers of the next module. The full model can be represented as a sequence of Swiss cheese slices where the probability of an event happening depends on a trigger and a concomitant alignment of holes in all slices (Fig. 2). ${ }^{17}$ The aggregation of all available patient data associated with imputation of missing information regarding factors relevant to each disease phase using probabilistic models allows drawing different management scenarios and ultimately should be able to predict outcomes for each of the scenarios. A Bayesian network graphical model was developed to visualize interactions and predictions dynamically for each disease phase (https://www.r-project.org/ conferences/useR-2009/slides/Klinger+Friedrich.pdf) (Fig. 3 ). The infrastructure so far allows for collecting data and managing biological samples to contribute in particular to the identification of genetic loci associated with the disease, ${ }^{19,20}$ assessing the aneurysm growth rate in the cohort of followed-up patients, ${ }^{10}$ assessing the predictive performance of an aneurysm rupture risk classifier based on location and size, ${ }^{2}$ PHASES score ${ }^{3}$ and cerebrovascular morphodynamic factors, ${ }^{6}$ and correlating clinical risk factors with histological characteristics of disease severity ${ }^{5,16}$ and associating biomechanical stress conditions with aneurysm

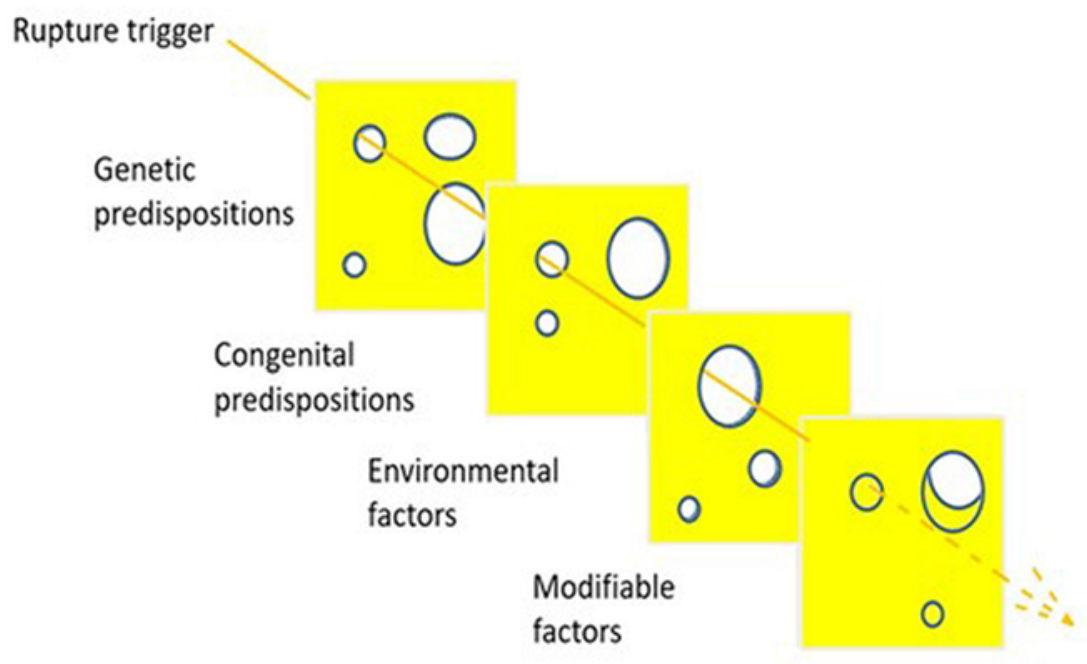

Aneurysm rupture

FIG. 2. The impact of multiple factors is visualized using a Swiss cheese model where different factors are each represented by a cheese slice with permissive traits (holes) and resistive traits blocking the effect of a transition trigger promoting the progression of the disease to another stage. 


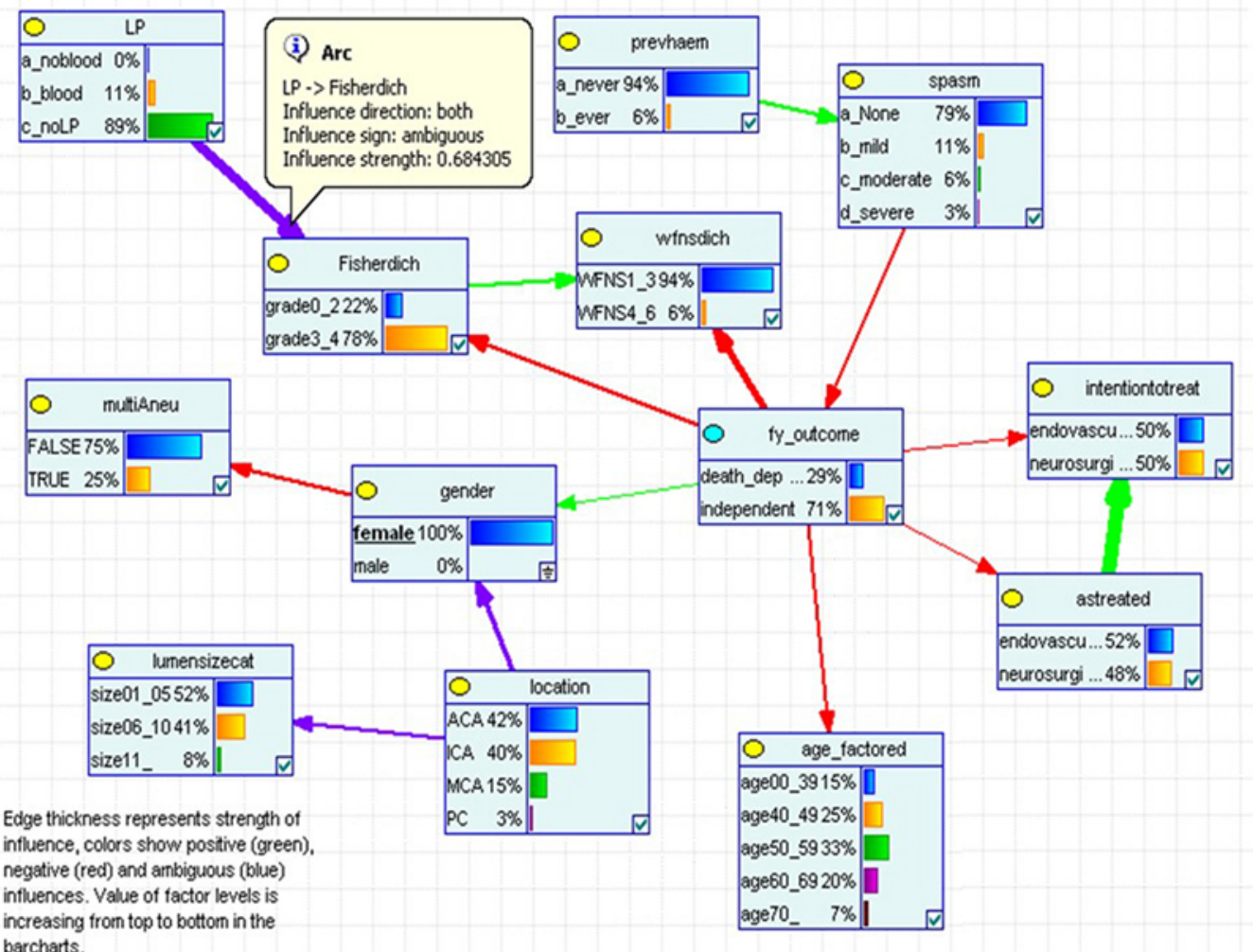

FIG. 3. Associations between factors and outcome can be visualized dynamically using Bayesian network models representing the direction and strength of associations between factors and distribution of probabilities regarding factor categories. In the example based on International Subarachnoid Aneurysm Trial study data, ${ }^{15}$ sex was fixed as female, and the distribution of cases in all other factors categories is dynamically displayed. The selection of any other category, i.e., an aneurysm location or size or both, will immediately result in the display of a new map with different arrows and distribution of cases in factor categories allowing the visualization of the different possible scenarios. Courtesy of Christoph M. Friedrich, developer of the rSMILE package.

initiation and growth. ${ }^{9,14}$ Analysis of the impact of different phenotypic and genetic factors on aneurysm initiation, multiplicity, and rupture is ongoing.

\section{Core Resources}

The community can share data by opening an account. The portal allows data to be deposited in association with meta-information regarding data collection conditions (purpose, protocol), definitions and data format used, and terms and conditions of data use, as well as the list of the contributors specifying their function and contribution period. Data can also be contributed using a decision support tool, providing information regarding risks associated with natural evolution and treatments or using a clinical integrated standardized reporting tool (AneuQuest) that supports structured data collection regarding clinical observations, decisions, treatments, and outcomes to be integrated in the hospital information system.

Once the data are submitted to the Aneurysm Data Bank (ADB), de-identification and encoding of the data are verified before being imported. Once imported, the data are harmonized to a standard information model that represents all dimensions relevant to the description of the disease and its management, the CRIM. An office within the ADB is dedicated to this harmonization activity, infra- structure maintenance, and upgrades according to information technology evolution, as well as to the management of data sharing according to agreements between investigators, coordinates collaborations, and manages data access rights.

Data access is gated by data user rights. Data can be accessed in two types of format: 1) aggregated data or 2) case-level data. According to user's rights, different services will be provided. Data providers will access two portals. One web portal will display aggregated data regarding their cohort basic characteristics, and the second portal will allow exploration and analysis of their own data. Data analysis is facilitated by integrated graphical user interfaces interfacing with the $\mathrm{R}$ free software environment (R Development Core Team, R Foundation for Statistical Computing) to allow easy statistical analysis.

Researchers interested in accessing harmonized data provided by different sources will have to claim access to data, providing a research protocol specifying aims, methods, and criteria for the inclusion or exclusion of cases as well as the list of data elements needed. Access to a data subset will be granted for a defined period of time and according to the protocol and upon agreement of data providers. Researchers will be able to upload case-based data for in-house analysis or have access to integrated graphical user interfaces interfacing with the $\mathrm{R}$ free software envi- 
ronment. Once a study is completed, the ADB will provide raw data, analysis protocol, and manuscript archiving services to researchers.

\section{Perspectives}

Efforts are now focused on consolidating and sustaining the structure of the ADB. The study of genetic factors influencing the development of the disease or having an impact on the consequences of bleeding requires the formation of very large cohorts, which is a great motivator for international collaborations. We hope to gradually federate a growing community.

As described above, the disease has a wide range of facets that, in order to be understood in the context of precision medicine, not only requires a large number of patients but also a fine and homogeneous description of the characteristics of the disease and its management. The current tool in its most basic version manages 13 variables, which is, for the moment, considered sufficient to start the genetic studies. Nevertheless, according to a large group of international experts (ISUIA), the list of factors that should be systematically assessed in order to decide whether or not to treat an intracranial aneurysm includes 35 factors. In its most extensive version, the inventory of potentially relevant information has more than 1000 factors and will necessarily expand further. At Geneva University Hospital, the collection of these data has been coupled with clinical documentation and the drafting of medical reports and letters. It is our desire to link other centers to a decision support tool for the management of intracranial aneurysms. Currently, a simple tool to estimate the risk of rupture of a specific aneurysm or calculate the balance of risks between observation and intervention on the basis of data from literature is available (http://iascore.swissneurofoundation.ch/). However, there is still a need for this tool to be accessible directly from the patient's electronic file.

The NeuroPoint Alliance (a nonprofit organization of the American Association of Neurological Surgeons established in 2008 with the mission improving quality of neurosurgical care through acquisition, analysis, and reporting of clinical data via registries and related studies) launched the Quality Outcome Database (QOD) neurovascular registry in $2014 .{ }^{1}$ Having similar aims, avenues to join efforts and collaborate will be explored.

Finally, clinical imaging and digital histopathology are powerful sources of high-quality information that can generate a digital replica of a patient (avatar) and opens the door to all kinds of biomechanical disease modeling and simulations. These technologies are already widely used in the fields of engineering. However, work is needed to improve the exchange of images and avatars.

\section{Conclusions}

The ADB infrastructure includes a set of elements that can serve as a platform for the exchange of information between the clinical community, researchers, health management bodies, and industry while supporting its management and offering a high level of security. The basic infrastructure can be used generically, and specific modules are adapted to the special needs of each particular organ or disease. It is designed to remain flexible and integrated with other existing or developing structures. The current purpose of the infrastructure is to demonstrate that it is possible to model a complex multifactorial disease and its management through collection, integration, and harmonization of data globally.

\section{Acknowledgments}

We would like to warmly thank the information technology team at the Hôpitaux Universitaire de Genève, in particular, Rodolphe Meyer, Nicolas Cassoni-Schoellhammer, Emmanuel Durand, David Cavin, Nicolas Roduit, and Marie-Laure Chardonnens as well as research nurse Nathalie Isidor without whom the ADB's infrastructure would not have become what it is and, above all, probably would not have survived the transition between the $@$ neurIST and AneuX project. We also thank Ioannis Xenarios, Jérôme Dauvillier and Robin Liechti at Vital-IT for their contribution to the implementation of the infrastructure.

The infrastructure was developed in the context of the @ neurIST project funded by the FP6 Framework Program of the European Commission Information Society Technologies IST (IST-2004-027703) and the AneuX project funded by the Swiss Initiative for Systems Biology SystemsX.ch and evaluated by the Swiss National Research Fund.

The contribution and trust in the project of the ISGC community needs to be warmly acknowledged as a strong driver and constant source of inspiration and motivation.

\section{References}

1. Asher AL, McCormick PC, Selden NR, Ghogawala Z, McGirt MJ: The National Neurosurgery Quality and Outcomes Database and NeuroPoint Alliance: rationale, development, and implementation. Neurosurg Focus 34(1):E2, 2013

2. Bijlenga P, Ebeling C, Jaegersberg M, Summers P, Rogers A, Waterworth A, et al: Risk of rupture of small anterior communicating artery aneurysms is similar to posterior circulation aneurysms. Stroke 44:3018-3026, 2013

3. Bijlenga P, Gondar R, Schilling S, Morel S, Hirsch S, Cuony $\mathrm{J}$, et al: PHASES score for the management of intracranial aneurysm: a cross-sectional population-based retrospective study. Stroke 48:2105-2112, 2017

4. Bijlenga P, Morita A, Ko NU, Mocco J, Morel S, Murayama Y, et al: Common data elements for subarachnoid hemorrhage and unruptured intracranial aneurysms: recommendations from the Working Group on Subject Characteristics. Neurocrit Care [epub ahead of print], 2019

5. Coen M, Burkhardt K, Bijlenga P, Gabbiani G, Schaller K, Kövari E, et al: Smooth muscle cells of human intracranial aneurysms assume phenotypic features similar to those of the atherosclerotic plaque. Cardiovasc Pathol 22:339-344, 2013

6. Detmer FJ, Fajardo-Jimenez D, Mut F, Juchler N, Hirsch S, Pereira VM, et al: External validation of cerebral aneurysm rupture probability model with data from two patient cohorts. Acta Neurochir (Wien) 160:2425-2434, 2018

7. Etminan N, Brown RD Jr, Beseoglu K, Juvela S, Raymond J, Morita A, et al: The unruptured intracranial aneurysm treatment score: a multidisciplinary consensus. Neurology 85:881-889, 2015

8. Ge H, Lv X, Jin H, Tian Z, Li Y, He H: The role of endovascular treatment in unruptured basilar tip aneurysms. Interv Neuroradiol 23:8-13, 2017

9. Geers AJ, Morales HG, Larrabide I, Butakoff C, Bijlenga P, Frangi AF: Wall shear stress at the initiation site of cerebral aneurysms. Biomech Model Mechanobiol 16:97-115, 2017

10. Gondar R, Gautschi OP, Cuony J, Perren F, Jägersberg M, Corniola MV, et al: Unruptured intracranial aneurysm 
follow-up and treatment after morphological change is safe: observational study and systematic review. J Neurol Neurosurg Psychiatry 87:1277-1282, 2016

11. Hui FK, Schuette AJ, Moskowitz SI, Spiotta AM, Lieber ML, Rasmussen PA, et al: Microsurgical and endovascular management of pericallosal aneurysms. J Neurointerv Surg 3:319-323, 2011

12. Kocur D, Zbroszczyk M, Przybyłko N, Hofman M, Jamróz T, Baron J, et al: Stand-alone coil embolization of anterior communicating artery aneurysms: efficacy and technical issues. Neuroradiol J 29:361-367, 2016

13. Lawton MT, Spetzler RF: Surgical management of giant intracranial aneurysms: experience with 171 patients. Clin Neurosurg 42:245-266, 1995

14. Machi P, Ouared R, Brina O, Bouillot P, Yilmaz H, Vargas MI, et al: Hemodynamics of focal versus global growth of small cerebral aneurysms. Clin Neuroradiol [epub ahead of print], 2017

15. Molyneux AJ, Kerr RSC, Yu LM, Clarke M, Sneade M, Yarnold JA, et al: International Subarachnoid Aneurysm Trial (ISAT) of neurosurgical clipping versus endovascular coiling in 2143 patients with ruptured intracranial aneurysms: a randomised comparison of effects on survival, dependency, seizures, rebleeding, subgroups, and aneurysm occlusion. Lancet 366:809-817, 2005

16. Morel S, Diagbouga MR, Dupuy N, Sutter E, Braunersreuther $\mathrm{V}$, Pelli G, et al: Correlating clinical risk factors and histological features in ruptured and unruptured human intracranial aneurysms: the Swiss AneuX Study. J Neuropathol Exp Neurol 77:555-566, 2018

17. Reason J: The contribution of latent human failures to the breakdown of complex systems. Philos Trans R Soc Lond B Biol Sci 327:475-484, 1990

18. Wiebers DO, Whisnant JP, Huston J III, Meissner I, Brown RD Jr, Piepgras DG, et al: Unruptured intracranial aneu- rysms: natural history, clinical outcome, and risks of surgical and endovascular treatment. Lancet 362:103-110, 2003

19. Yasuno K, Bakırcıŏ̆lu M, Low SK, Bilgüvar K, Gaál E, Ruigrok YM, et al: Common variant near the endothelin receptor type A (EDNRA) gene is associated with intracranial aneurysm risk. Proc Natl Acad Sci U S A 108:19707-19712, 2011

20. Yasuno K, Bilguvar K, Bijlenga P, Low SK, Krischek B, Auburger G, et al: Genome-wide association study of intracranial aneurysm identifies three new risk loci. Nat Genet 42:420-425, 2010

\section{Disclosures}

The authors report no conflict of interest concerning the materials or methods used in this study or the findings specified in this paper.

\section{Author Contributions}

Conception and design: Bijlenga, Rüfenacht. Acquisition of data: all authors. Analysis and interpretation of data: Bijlenga, Hirsch, Rüfenacht. Drafting the article: Bijlenga. Critically revising the article: Morel, Hirsch, Schaller, Rüfenacht. Reviewed submitted version of manuscript: Morel, Hirsch, Schaller, Rüfenacht. Approved the final version of the manuscript on behalf of all authors: Bijlenga. Administrative/technical/material support: all authors. Study supervision: Bijlenga.

\section{Correspondence}

Philippe Bijlenga: Hôpitaux Universitaires de Genève, Geneva, Switzerland.philippe.bijlenga@hcuge.ch. 